\title{
La Comprensión y el Involucramiento con el Arte desde la Perspectiva de la Segunda Persona
}

\author{
[Understanding and Involvement with Art from the Second-Person Perspective]
}

\author{
Diana I. Pérez]
}

Resumen: El trabajo muestra que la perspectiva de segunda persona de la atribución mental (Pérez 2013 y Pérez y Gomila 2021) puede contribuir a iluminar nuestra comprensión e involucramiento con el arte. Una forma canónica de comprender nuestro involucramiento con el arte pone el foco en la noción de empatía, que también suele ser considerada una noción central para dar cuenta de nuestro acceso a las otras mentes. En la primera parte del trabajo hago un breve repaso de esta noción y la conecto con trabajos recientes en el ámbito de la filosofía del arte en los que se adopta una teoría simulacionista de nuestra comprensión de las mentes y de la experiencia estética. En segundo lugar, muestro las limitaciones que estas nociones (empatía y simulación) tienen para dar cuenta de nuestra comprensión e involucramiento tanto con los demás seres humanos como con el arte y expongo brevemente los lineamientos centrales de la perspectiva de segunda persona de la atribución psicológica. Finalmente, muestro por qué la perspectiva de segunda persona es más adecuada para dar cuenta de las múltiples y multifacéticas experiencias que surgen con el arte y las actividades artísticas.

Palabras clave: Empatía. Simulación. Postcognitivismo. Cognición social. Expresión.

\begin{abstract}
This paper shows that the second-person perspective of mental attribution (Pérez 2013 and Pérez and Gomila 2021) can contribute to illuminate our understanding and involvement with art. A canonical way of understanding our involvement with art focuses on the notion of empathy, which is also considered a central notion to account for our access to other minds. In the first part of this paper, I briefly review this notion and I connect it with recent works in the field of philosophy of art in which a simulationist theory is adopted, both to account for our understanding of other people's minds and for understanding aesthetic experiences. Secondly, I show the limitations that these notions (empathy and simulation) have to account for our understanding and involvement both with other human beings and with art and I briefly expose the central thesis of the second person perspective of psychological attribution. Finally, I show why the second-person perspective is more adequate to account for the many and multifaceted experiences that arise with art and artistic activities.
\end{abstract}

Keywords: Empathy. Simulation. Postcognitivism. Social Cognition. Expression.

Profesora Titular. Departamento de Filosofía. Facultad de Filosofía y Letras de la Universidad de Buenos Aires. Investigadora Principal IIF-SADAF-CONICET. E-mail: dperez@filo.uba.ar. ORCID: https://orcid.org/0000-0002-6185-7986. 
En este trabajo me propongo mostrar que la perspectiva de segunda persona de la atribución mental puede contribuir a iluminar nuestra comprensión e involucramiento con el arte 11 Como explicaré más adelante, la comprensión de las mentes humanas y el involucramiento con los otros seres humanos son fenómenos que se entretejen con nuestra experiencia estética desde los inicios de las interacciones sociales. Así, la idea detrás de este trabajo es que la comprensión e involucramiento con el arte y la comprensión e involucramiento con otros seres humanos son fenómenos relacionados, por lo que estudiarlos paralelamente puede ofrecer frutos tanto para avanzar en la filosofía de la mente como en la filosofía del arte. En trabajos anteriores (Pérez 2013 y Pérez y Gomila 2021) he desarrollado una propuesta dentro del ámbito de la cognición social desde una perspectiva postcognitivista: la perspectiva de segunda persona de la atribución mental. Es mi intención proyectar esta propuesta postcognitivista a las consideraciones filosóficas relativas a la experiencia estética y a nuestro involucramiento en actividades artísticas. En este trabajo asumiré una comprensión muy amplia de "arte" incluyendo no sólo las canónicas obras de arte de nuestra cultura occidental (una pintura de Picasso, una sinfonía de Beethoven, un cuento de Borges, etc.) sino también muchas otras manifestaciones y actividades artísticas como pinturas rupestres, garabatos de niños, canciones tribales alrededor de una fogata o recitales de rock, comics, mangas y cuentos infantiles. Nuestro involucramiento con estas variadas formas de manifestaciones musicales, plásticas, ficcionales, etc., son ubicuas en nuestras vidas, y constituyen nuestra identidad humana (aunque esta última tesis no la voy a defender aquí en detalle).

La idea de que nuestra comprensión e involucramiento con otras mentes es un fenómeno relevante para dar cuenta de nuestra experiencia estética no es, sin embargo, nueva. En efecto, una forma canónica de comprender nuestro involucramiento con el arte pone el foco en la noción de empatía, que también suele ser considerada una noción central para dar cuenta de nuestro acceso a las otras mentes. Es por eso que comenzaré este trabajo haciendo un breve repaso de esta noción y la conectaré con trabajos recientes en el ámbito de la filosofía del arte en los que se adopta una teoría simulacionista de nuestra comprensión de las mentes y del arte. En segundo lugar, mostraré las limitaciones que estas nociones (empatía y simulación) tienen para dar cuenta de nuestra comprensión e involucramiento tanto con

\footnotetext{
${ }^{1}$ Utilizaré en este artículo la expresión "experiencia estética” como sinónimo de la experiencia de comprensión e involucramiento que se da en presencia de canónicas obras de arte, y que se extiende a situaciones no canónicas también. No busco explicar aquí los rasgos que la tradición involucra en esta expresión, a saber que se trate de una experiencia con un carácter inmediato, y/o desinteresado y/o que involucre la idea de belleza.
} 
los demás seres humanos como con el arte y expondré brevemente los lineamientos centrales de la perspectiva de segunda persona de la atribución psicológica. Mostraré finalmente, por qué la perspectiva de segunda persona es más apta para dar cuenta de las múltiples y multifacéticas experiencias que surgen con el arte y las actividades artísticas.

I.

La noción de empatía, (y la de simpatía tal como surge en el siglo XVIII a partir de la obra de autores como David Hume y Adam Smith), tiene dos roles teóricos. En primer lugar tiene la función de dar cuenta de un vinculo psicológico especial existente entre los seres humanos, que es justamente la raíz de ciertas discusiones contemporáneas acerca de nuestras capacidades para captar los contenidos de los estados mentales de nuestros congéneres. El segundo aspecto se relaciona con nuestra respuesta moral a otros seres humanos, que de alguna manera esta basada en nuestra forma empática de conectarnos con nuestros congéneres..$^{2}$ Hacia fines del siglo XIX y principios del XX Theodor Lipps adoptó la empatía (o "proyección sentimental") como eje de su explicación de la experiencia estética. Su presentación de esta idea se da en los siguientes términos:

Cuando contemplo en otro individuo un gesto de tristeza entra en acción en mí el instinto imitativo. La percepción e interpretación del gesto ajeno despierta en mí, sin saber yo cómo, dicha tendencia... esta tendencia vivida en mí, es la misma que antes se daba inmediatamente en el afecto de la tristeza. Una parte de aquel hecho psíquico está de nuevo allí, en mí. Y... nace en mí la tendencia a caer en el estado afectivo, otras veces experimentado o sentido, y correspondiente a dicha tendencia....En suma, todo esto quiere decir que yo, en la percepción del gesto ajeno, siento inmediatamente el afecto tal como en él se anuncia. (Lipps, 1924, pp. 2526, mis itálicas)

De acuerdo con Lipps, este mismo mecanismos psicológico que me permite acceder a las mentes ajenas inmediatamente, imitativamente, basándome en su corporalidad expresiva, es el que está a la base de la experiencia estética. Así, con la expresividad de las obras de arte se despiertan en nosotros estos mismos mecanismos imitativos que nos

\footnotetext{
${ }^{2}$ Voy a dejar de lado en este trabajo la dimensión moral de esta noción, y me voy a centrar en la noción de empatía como forma de entendernos los unos a los otros como seres con mentes, y de dar cuenta de nuestra relación con el arte, y la vinculación entre ambas dimensiones.
} 
llevan a proyectar en la obra nuestros propios sentimientos, atribuyéndoselos a ella.

La empatía es, entonces, este proceso de imitación o de resonancia interna que nos lleva a imitar movimientos y expresiones que percibimos en otros seres humanos, a experimentar los sentimientos del otro como propios y/o a proyectar nuestros sentimientos en el otro (Coplan y Goldie 2011). En el caso del arte, involucra la idea de que las obras de arte de alguna manera provocan o dan lugar a las mismas respuestas en nosotros que las que producen los movimientos y expresiones del cuerpo de los otros seres humanos y/o que proyectamos nuestras cualidades subjetivas en las obras de arte. Es importante remarcar algo que está detrás de todas las caracterizaciones de empatía: la coincidencia o resonancia o imitación de los estados mentales del otro o de la obra de arte. Si hay empatía, hay una coincidencia entre mis estados mentales (los estados mentales del sujeto, aquellos a los que se accede en primera persona) y los estados mentales del otro (la otra persona, la obra de arte).

En los últimos 40 años, sin embargo, la empatía fue dejada de lado a la hora de buscar una explicación de las formas en las que comprendemos las mentes humanas. En efecto, durante las últimas dos décadas del siglo XX hubo dos grandes apuestas teóricas en la marco de la filosofía de la mente y de la psicología: la teoría de la teoría y la teoría de la simulación. La primera sostiene que nuestra forma de conocer las mentes humanas no difiere de la forma habitual en la que tenemos accesos a cualquier dominio de conocimiento: tenemos experiencias, observamos el mundo, formamos hipótesis generales acerca de las sucesiones de eventos, incluyendo tanto los físicos como los psicológicos, y así acumulamos un conocimiento general de cada dominio físico, biológico, psicológico, etc. Para entender las acciones ajenas, entonces, simplemente aplicamos nuestro conocimiento general acerca de las mentes humanas (la psicología folk) y deducimos de los principios generales el caso particular. Por su parte, la teoría de la simulación parte del caso particular de primera persona. Tomando al propio sujeto como punto de partida se realizan proyecciones al caso cuya conducta quiere comprender, proyectándose en el lugar del otro - en alguna versiones al lugar físico, en otras a la situación psicológica del otro- para imaginar cómo hubiera actuado estando allí.3

A estas dos propuestas desarrolladas en el marco de posiciones cognitivistas tanto en psicología como en filosofía se le han contrapuesto, en los últimos 20 años, nuevas teorías que adoptan un marco postcognitivista, como el interaccionismo (Gallagher 2001), el enac-

\footnotetext{
${ }^{3}$ Véase Pérez 2013 para una presentación crítica de estas dos alternativas teóricas.
} 
tivismo (De Jaegher Di Paolo 2007) y la perspectiva de segunda persona (Gomila 2001, Scotto 2002, Pérez y Gomila 2021), que es la que me interesa considerar en este trabajo. $4^{4}$

Por su parte, en el ámbito de la filosofía del arte, aquellos que buscaron rescatar la conexión entre las teorías que dan cuenta de nuestra comprensión de las mentes humanas y la estética, han adoptado ampliamente la teoría simulacionista (Currie 1995, Currie y Ravencroft 2002, Walton 2015). Hay dos razones por las que la teoría de la simulación ganó adeptos dentro del ámbito de la filosofía del arte. En primer lugar, la teoría de la simulación permite entender la comprensión de las mentes en términos de la proyección de mi mente hacia el otro, o sea suponiendo una coincidencia de mis estados mentales con los del otro tal como la empatía supone. Es decir, la teoría simulacionista de alguna manera le pone ropajes nuevos a la vieja teoría de la empatía. $5^{5}$ En segundo lugar, la teoría simulacionista ubica a la imaginación como facultad psicológica subyacente al proceso de simulación, en coincidencia con las teorías del arte que también ponen en primer plano el proceso imaginativo que nos permite proyectarnos en la obra de arte, y que subyace, en general al juicio estético.

Es importante destacar, además, que a partir de los últimos años del siglo pasado este proceso de simulación parece haber encontrado una base empírica en el sistema de neuronas espejo (Gallese et al 1996, Rizzolatti et al 1996, Gallese Goldman 1998) y desde hace unos 10 años - en el marco de los ampliamente desarrollados estudios en el ámbito de la neuroestética - Gallese se ha dedicado a estudiar cómo funciona nuestro sistema de neuronas espejo en el proceso de precepción de obras de arte, en nuestra experiencia estética, a través de mecanismos que denomina de "simulación corporizada" (Freedberg y Gallese 2007, Gallese 2010, 2017). Así, tanto la teoría de la simulación como su aplicación a la experiencia estética parecen contar con apoyo empírico en las neurociencias.

En este trabajo voy a dejar de lado las consideraciones acerca del rol de la facultad de la imaginación en general tanto en lo que respecta a la comprensión de las otras mentes como al arte. Creo que la imaginación puede jugar un rol, pero no es el mecanismo básico

\footnotetext{
${ }^{4}$ La expresión "perspectiva de segunda persona" es ambigua. Por un lado la usamos para referirnos a una posición teórica que adopta como punto de partida las interacciones entre dos individuos para dar cuenta de las formas más básicas de acceso a las mentes humanas. Esta perspectiva teórica la hemos desarrollado en detalle en Pérez y Gomila 2021 y es a ella a la que me referiré en este trabajo con esta expresión, a menos que se indique lo contrario. Pero, por otro lado, la expresión "perspectiva de segunda persona" también puede usarse para contrastarla con la perspectiva de tercera persona que adopta una persona para entender a otro individuo, cuando lo hace desinvolucradamente, externamente, observacional e inferencialmente, - a diferencia de lo que pasa cuando adopta la perspectiva de segunda persona, en donde hay un involucramiento afectivo y un acceso directo, no inferencial a la mente del otro, y a la perspectiva de primera persona, cuando accedemos directa y no inferencialmente a nuestros propios estados psicológicos.

${ }^{5}$ Goldman 2006.
} 
en nuestras formas de comprensión de las mentes. Puede que la imaginación esté involucrada en nuestra comprensión e involucramiento con el arte, para muchos la empatía supone un ejercicio imaginativo, pero esto no es así para todos los filósofos del arte (véase, por ej. Walton 2015 cap. 1). Así, dejaré a un lado la discusión acerca del rol de la imaginación en general en la apreciación del arte, y me centraré exclusivamente en las dificultades que la empatía y la teoría de la simulación tienen para dar cuenta tanto de las mentes ajenas como de la experiencia estética.

II.

Hay una extensa literatura en la que se discuten las limitaciones de la teoría de la simulación para dar cuenta de nuestro acceso a las mentes ajenas. ${ }^{6}$ Yo quisiera centrarme en esta ocasión en dos problemas fundamentales que comparten la simulación con la empatía, y que creo que resultan una dificultad tanto para dar cuenta de las mentes ajenas como de la experiencia estética. El primer problema que quiero mencionar es el que resulta de tomar como punto de partida la primera persona. En la mayoría de las versiones de la simulación y de la empatía se supone el acceso introspectivo a la propia men- te y sus contenidos, mecanismo que es anterior, más básico y fiable que el acceso a las mentes ajenas, que siempre supone algún tipo de proceso inferencial a partir de la primera persona. En sus versiones más extremas, esto supone la presencia de "conceptos fenoménicos" para formular juicios acerca de la propia experiencia y proyectarlos al otro (Goldman 1993) o a la obra de arte (Walton 2015). En mi opinión la estrategia de los conceptos fenoménicos es inviable (Pérez 2011, 2014). Y si la introspección no hace uso de estos conceptos fenoménicos o de primera persona, entonces antes de iniciarse el proceso de simulación o empático es necesario tener a disposición un conjunto de conceptos psicológicos que sean aplicables tanto a la primera como a la tercera persona, por lo tanto no se puede pensar a este mecanismo que parte de la primera persona como el más básico. La perspectiva de segunda persona, por el contrario, supone que es en las interacciones de segunda persona (yotú) donde se dan las primera atribuciones psicológicas porque es en ellas donde se adquieren los distintos conceptos psicológicos que hacen posibles estas atribuciones, conceptos que implican al mismo tiempo la habilidad de auto- y hetero-atribución (Pérez 2013, Pérez y Gomila 2021).

Sin embargo, la dificultad central y

\footnotetext{
${ }^{6}$ Un locus clásico de estas críticas abarcando las diversas versiones de la teoría de la simulación es Gallagher 2008. En Pérez 2013 cap. 4 repaso diversas versiones de esta teoría y sus dificultades.
} 
más importante que veo para usar ideas como la de simulación o empatía tanto para dar cuenta de nuestra comprensión de las otras mentes como para de la comprensión e involucramiento con el arte, es que no parece razonable sostener que sea necesaria la identidad de experiencias para que comprendamos, apreciemos y disfrutemos de una obra de arte, ni para entender las mentes de nuestros congéneres. En efecto, muchas veces logramos una comprensión exitosa de las mentes de las personas con las que interactuamos, sin por ello coincidir en nuestro pensamientos, sensaciones o emociones. Así, la perspectiva de segunda persona de la atribución mental es una mejor opción para dar cuenta de los fenómenos que nos ocupan, porque tiene un espectro más amplio de posibilidades dado que acepta como caso particular aquel de la coincidencia entre los estados mentales de los individuos (o del individuo y la obra de arte), pero acepta la posibilidad de nocoincidencia.

Permítanme ahora resumir brevemente qué voy a entender aquí por "perspectiva de segunda persona" de la atribución mental como alternativa a otras teorías relevantes acerca de cómo comprendemos las mentes de las personas. En el próximo apartado intentaré aplicar estas ideas al caso de nuestra experiencia con las obras de arte.
La perspectiva de segunda persona (Gomila 2002, Scotto 2002, Pérez 2013, Pérez y Gomila 2021) es una propuesta teórica dentro del ámbito de la cognición social que asume la visión postcognitivista de la mente. 7 El punto de partida que se propone no es el del individuo aislado, que conoce sus propios estados mentales, sino el de las interacciones de segunda persona, aquellas situaciones en las que dos seres humanos se encuentran cara a cara, cuerpo a cuerpo y se comprenden y perciben como seres humanos que realizan acciones intencionales, que se emocionan y que piensan como tales. La tesis central de la perspectiva de segunda persona es que este tipo de interacciones está mediada por un tipo característico de atribuciones psicológicas, denominadas atribuciones de segunda perso$n a$, que son atribuciones automáticas, prácticas, implícitas, transparentes, recíprocamente contingentes y dinámicas. La perspectiva de segunda persona asume que este tipo de atribuciones son las más básicas (filogenética, ontogenética y conceptualmente). Pero no niega que haya otras perspectivas de atribución psicológica (en el sentido de otros puntos de vista - ver nota 4- ajenos a la interacción de segunda persona en los que se hacen atribuciones psicológicas, podríamos denominarlas perspectivas de primera y tercera persona), por el

\footnotetext{
${ }^{7}$ Suele resumirse la posición postcognitivista a través de la 4E/5E: la idea de una mente corporizada/encarnada (embodied), extendida, situada (embedded), enactiva y emotiva.
} 
contrario se afirma que son necesarias las tres perspectivas para explicar con exhaustividad las complejas formas de comprensión mutua que desplegamos los seres humanos, tanto cuando nos encontramos en interacciones de segunda persona como cuando estamos solos, o somos simplemente observadores no participantes de las acciones ajenas.

El desarrollo de la perspectiva de segunda persona ha encontrado un fértil territorio de inspiración en las investigaciones en el ámbito de la psicología del desarrollo que se ocupa de la infancia temprana, dado que las interacciones adulto-bebé constituyen un tipo de interacción paradigmático de segunda persona (Reddy 2008). En estas interacciones se verifican formas de involucramiento y de comprensión mutua que no dependen de la posesión de un lenguaje público, ni suponen la realización de complejas atribuciones de estados mentales con contenido proposicional. Por el contrario, en estos casos se realizan atribuciones de mentalidad directamente, percibiendo la expresión corporal, facial, sonora, etc. del otro. En nuestra conducta interactiva manifestamos nuestro placer, disgusto, alegría, reaccionando tanto por la forma en la que el otro se dirige a nosotros como por cómo nos afectan los objetos y eventos del mundo compartido. La comprensión directa que tenemos de quien está enfrente nuestro nos afecta y produce una respuesta inmediata, no reflexiva, automática, afectiva e intencional, una reactividad emocional ante la expresión que nos muestra los estados psicológicos del otro. Dado que las emociones y estados afectivos en general son la puerta de entrada y el sostén de estas interacciones y las constitutivas comprensiones mutuas, el involucramiento afectivo es un pilar fundamental de las interacciones de segunda persona.

Sin embargo, hay mucho más en juego en nuestras interacciones con los demás que estas atribuciones de segunda persona y estos intercambios comunicativos. La adquisición de pautas culturales y normas propias de la sociedad en la que vivimos dan forma a las complejas maneras de interacción humana y la adquisición de un lenguaje público nos permite atribuir estados mentales más complejos, con contenido proposicional, y comprender con más fineza las intenciones y acciones ajenas (y las propias). Es en las interacciones tempranas de segunda persona que se da la ocasión para la adquisición de muchas de las habilidades cognitivas que desplegamos a lo largo de nuestra vida, no sólo adquirimos los conceptos psicológicos que nos permiten comprender las mentes humanas, sino que también adquirimos la lengua materna, así como una enorme cantidad de pautas sociales que regulan nuestras acciones y que nos permiten -a medida que vamos creciendo- realizar tareas tan variadas como cocinar, danzar, participar de competencias de nado, andar en bicicleta, cantar, tocar instrumentos e in- 
volucrarnos en las diversas prácticas artísticas de nuestra cultura. Una vez adquiridas (en el contexto de las interacciones de segunda persona) las diversas habilidades que nos permiten participar en diversas prácticas sociales, podemos usarlas más allá de los contextos de interacción. Podemos autoatribuirnos estados mentales, cocinar o cantar solos; y podemos hacer atribuciones psicológicas a individuos con los que no estamos interactuando; a individuos pasados y futuros; alejados de nosotros en el espacio; individuos ficticios, etc., así como podemos escuchar un disco de Freddy Mercury, o ver una grabación de Rudolf Nureyev bailando el "El casacanueces". También percibimos esos patrones expresivos en las nubes del cielo, en las hojas caídas del otoño que el viento mueve en la vereda, en el canto de los pájaros, y en las obras de arte que vemos en un museo. Pero, por más que a lo largo de nuestra vida podamos adquirir una enorme cantidad de novedosas capacidades cognitivas, estas formas iniciales de comprensión de los otros en la interacción están siempre presentes. En efecto, las formas de interacción adultas siguen teniendo a la base las formas de interacción de segunda persona que describí más arriba, entretejidas con normatividades varias que hacen posibles formas más complejas de interacción (pensemos en la variedad de capacidades en juego cuando estamos en un dialogo cara a cara con alguien, o cuando dos músicos to- can juntos una pieza, o cuando bailamos una milonga con nuestra pareja).

Pero además, una vez adquiridas las habilidades cognitivas relevantes, podemos alejarnos de la actitud participativa, tomar distancia y evaluar fríamente las acciones ajenas. A veces adoptamos un punto de vista más universal que nos aleja del individuo concreto que tenemos delante y nos preguntamos por la corrección de sus acciones (esto es, por su validez universal); a veces adoptamos la actitud del crítico tratando de valorar las acciones ajenas, una pieza de baile, una obra de arte. En general, adquirir pautas culturales nos permite comprender que ciertas acciones están de acuerdo con la norma, y que otras acciones se alejan de ella: que una emisión lingüística es semánticamente significativa o un sinsentido, que una acción está de acuerdo con las normas de cortesía de nuestra comunidad o que resulta grosera, que un cuadro o melodía se adecua o se aleja de las normas propias del movimiento artístico al que pertenece, etc.

En nuestros encuentros tempranos con otros - en nuestra infancia temprana- participamos de los patrones que subyacen a las prácticas artísticas de la cultura en la que estamos inmersos. Los estudios en el ámbito de la psicología de desarrollo han conectado las formas de interacción adulto-bebé (lo que he denominado "interacciones de segunda persona") con las diversas formas de arte. Algunas de las ideas 
en este ámbito son las siguientes. Trevarthen, un pionero en el desarrollo de las ideas de intersubjetividad que dan sustento a la perspectiva de segunda persona, ha desarrollado recientemente la idea de musicalidad comunicativa (Malloch and Trevarthen 2009, 2018) con el fin de describir las interacciones tempranas, destacando el rol que ciertos rasgos musicales como el contorno melódico, el ritmo, etc. tienen en los intercambios intersubjetivos. Estos patrones rítmicos pueden ser observados en los ritmos de succión del bebé, en los intercambios de turnos en las protoconversaciones, en las características del habla dirigida al bebé ("Baby talk" o "Motherese"), un lenguaje simplificado caracterizado por un tono alto, con un rango tonal más amplio, entonación exagerada (Fernald 1985) y en las actuaciones que los adultos realizan hacia los bebés. Las actuaciones dirigidas a los bebés han sido estudiadas tanto desde la música como desde la danza (Español y Shifres 2015), porque en ellas se coordinan los contornos melódicos y kinéticos, y exhiben una coordinación multimodal también presente en la danza. Asimismo, es de destacar la existencia de las canciones de cuna presentes en todas las culturas, en las que madres y padres cantan a los niños adoptando patrones sonoros específicos (Trehub et al 1993, 1997). Otro ámbito de prolífico estudio en la psicología del desarrollo concierne a la relación entre el juego infantil y el arte. (Véase Espa- ñol y Pérez 2015).

En resumen: desde la perspectiva de segunda persona, el acceso a las mentes del otro en condiciones ecológicas de interacción cuerpo a cuerpo se da de forma directa, perceptual, depende de los patrones expresivos del individuo $y$ permite acceder a sus estados mentales que no necesariamente coinciden con los del otro sujeto que está interactuando. Estas interacciones además están fundadas en el involucramiento afectivo que se da entre los individuos $y$ en las reacciones emocionales expresadas en la corporalidad que son centrales para que la interacción fluya sin tropiezos. Los patrones expresivos presentes en estas interacciones pueden ser analizadas atendiendo a sus cualidades estéticas, que se encuentran presentes ya en las interacciones de segunda persona en la temprana infancia.

\section{III.}

Creo que es una propuesta interesante aplicar los mecanismos que usamos para entender las mentes humanas al caso del arte. Por un lado, porque las obras de arte son el resultado de acciones humanas, o son -en el caso de las artes performativas- acciones humanas ellas mismas. Como sostiene Gell $(1998 / 2016)$ es en el contexto de una teoría de la agencialidad humana que las obras de artes y las actividades artísticas en general cobran sentido. Co- 
mo dije en I., la idea original de Lipps (y otros autores posteriores) fue la de aplicar la empatía -entendida como mecanismo de comprensión de las otras mentes- a la obra de arte, para dar cuenta de la naturaleza de nuestra experiencia estética. Sin embargo, como expuse en II. la perspectiva de segunda persona parece una mejor teoría que la empatía o la teoría de la simulación para dar cuenta de los mecanismos de comprensión de las otras mentes, por lo que mi propuesta es aplicar esta perspectiva para dar cuenta de nuestra comprensión e involucramiento con el arte y en prácticas artísticas. 8

Hay antecedentes de exploración de esta idea en el contexto de formas de arte específicas. En efecto, Gomila 2009 se ha ocupado de la segunda persona y la música y más recientemente Martínez y su grupo de investigación (Martínez, 2021) han estudiado el rol de las atribuciones de segunda persona entre músicos de diversos géneros (jazz, orquesta clásica, tango, etc.). Gomila 2013 ha propuesto estudiar el rol de la segunda persona en el contexto de las representaciones teatrales. Por su parte, en un trabajo reciente Reddy avanza un paso más, defendiendo la idea de que la perspectiva de segunda persona también es importante para dar cuenta de la interacción en el contexto artístico con materiales, analizando el caso del artista de cerámica y el bloque de cerámica, quienes, según sostienen muchos artistas, mantienen un dialogo yo-tú a la hora de crear la pieza (Brinck y Reddy 2020). En lo que sigue intentaré dar un argumento más general en favor de la importancia de la adopción de la perspectiva de segunda persona para dar cuenta de nuestra experiencia estética. Sostendré la importancia de atender a las relaciones de segunda persona en las diversas formas de arte, revisando las posibles interacciones relevantes en este aspecto. No voy a sostener que con estas consideraciones disponemos de todos los elementos relevantes para dar cuenta de la experiencia estética. Por el contrario, creo que la segunda persona sola no alcanza, así como no alcanza para dar cuenta de las múltiples y complejas atribuciones de estados psicológicos que hacemos los humanos, como sostuve arriba. Sin embargo, creo que la perspectiva de segunda persona permite prestar atención a ciertos elementos presentes en la relación que tenemos con el arte que no han sido particularmente desatacados en la literatura.

Como se expuso más arriba la empatía (y la simulación) suponen la coincidencia de estados mentales entre quien aprecia el arte y la obra. La perspectiva de segunda persona no supone tal coincidencia. Por el contrario, considera al otro individuo en la interacción como

\footnotetext{
${ }^{8}$ Considero que la perspectiva de segunda persona es iluminadora tanto para comprender nuestra experiencia como espectadores o apreciadores de arte, como nuestra experiencia como performers, esto es como artistas involucrados en una práctica, por ejemplo, de ejecución musical, danza, actuación, incluso pintura y escritura.
} 
un otro, cuyos estados mentales se perciben directamente y se reacciona ante ellos, sin por ello coincidir; por el contrario, en general, son diferentes. Por ejemplo, veo a mi amiga con su corazón roto y mi reacción es de comprensión y acompañamiento, no se me rompe a mi el corazón, ni imagino como sería tener el corazón roto para brindarle mi apoyo y contención; veo que le pegan a un niño y no siento dolor, sino ira por la injusticia cometida, etc. El caso de la coincidencia es un caso especial contemplado dentro de la perspectiva de segunda persona, pero no el único ni el más usual. Lo mismo, sostengo, ocurre con las obras de arte. Y la razón es bastante simple: en el caso de las obras de arte no hay nunca una única mente que comprender y con la cual involucrarse. Por el contrario, una de las cosas interesantes en el caso del arte es que hay una pluralidad de "otros" con los que interactuamos. Por supuesto, hay grandes variaciones en lo relativo a los "otros" involucrados dependiendo de la forma de arte de la que hablemos. En lo que sigue, voy a mencionar diversas interacciones de segunda persona relevantes en el caso del arte, y procuraré dar ejemplos. No intento sostener la idea de que todas estas formas de interacción están presentes en todas las formas de arte. Me limitaré a dar un listado de los diversos tipos de interacciones posibles.

En primer lugar, el apreciador se involucra y comprende a los personajes de la obra si los hay, sea en una nove- la, una obra de teatro, un cuadro, una sinfonía -incluso los personajes pueden ser los instrumentos. En efecto, es parte constitutiva de nuestro involucramiento con las obras de arte narrativas que reaccionemos y nos involucremos con las aventuras y desventuras de los personajes de la obra. No sólo necesitamos comprender lo que les pasa para poder seguir la trama de la obra, sino que a través de la obra nos involucramos afectivamente con ellos, los personajes nos importan, deseamos que tengan éxito o fracasen, que se enamoren o que el asesino sea descubierto. La perspectiva de segunda persona puede dar cuenta de una manera sencilla de este tipo de involucramiento, ya que como dije en el apartado anterior puede dar cuenta no sólo de la relación que tenemos con otro con el que interactuamos realmente, sino también permite explicar como trascendemos el aquí y ahora para atribuir estados mentales e involucrarnos con personajes pasado, o ficticios. La perspectiva de segunda persona destaca el rol central de nuestro involucramiento afectivo como base para la cognición social.

En segundo lugar, el apreciador está en relación con el performer: con el actor, como diferente del personaje; el músico que ejecuta la obra en frente a nosotros, que puede no ser el autor de la pieza ni -obviamente- el personaje de la pieza musical, etc. Es importante mencionar que esta relación en algunas formas de arte es muy distante. Las formas 
canónicas de consumo de arte en la cultura occidental suelen involucrar situaciones en las que el espectador es muy pasivo, como la sala de teatro o concierto (el extremo más claro es el cine, donde no existe interacción alguna con los actores). 9 Sin embargo, hay formas de arte mucho más interactivas: el teatro participativo, el concierto de rock (en el que el artista interactúa y habla con el público), la sala de café concert o el stand-up. En muchas formas de arte el artista involucra al apreciador y lo vuelve parte de la obra. La perspectiva de segunda persona tiene como foco central de su teoría este tipo de interacciones dinámicas y mutuas, y destaca el rol que nuestra comprensión de la mente del otro tiene en estas interacciones.

En tercer lugar, hay otras personas involucradas en las obras de arte a las que tenemos un acceso mucho menos directo, más inferencial, hipotético, por ejemplo el narrador en una obra narrativa (que puede no ser ni el autor ni un personaje). Y también se puede considerar como otra persona al punto de vista implícito en la obra (el punto de vista desde el cual se ve el cuadro), la "persona" de la música, el "autor implicado" en una novela, etc. Se trata de un elemento que el lector/espectador construye al contemplar la obra, y en ese sentido no es un "otro" auténtico, en el sentido de que de alguna manera no es un individuo independiente del apreciador. Sin embargo, es un individuo más en juego a la hora de comprender una obra de arte, que a veces expresa el punto de vista de este ser hipotético, independiente del artista de carne y hueso que creó la obra, de los personajes que incluye, y de los artistas que le dan vida a la obra.

En cuarto lugar, toda obra de arte es una creación humana, por lo que toda obra involucra una intencionalidad o agencialidad que en primer lugar hizo posible la existencia de ese objeto. En la cultura occidental la autoría artística es central, por eso hablamos de un Miró, o del estilo inconfundible de Beethoven. Pero esto no ocurre en todas las culturas, en las que quién sea concretamente el artista es irrelevante, tal como ocurre con el arte religioso de la edad media: la intencionalidad es relevante, no la identidad del autor. Lo mismo ocurre con el arte de la Melanesia a cuyo estudio se dedica Gell. Así, el autor no siempre está presente, pero siempre está implícito: una obra de arte es algo que un humano hizo, o que un humano puso en un lugar para instituirlo como tal. Muchas veces el conocimiento que podemos tener de la identidad e

\footnotetext{
${ }^{9}$ Nótese, sin embargo, que muchas veces el montaje del film nos induce a sentirnos en interacción con los personajes. Un caso claro es en aquellas escenas en que dos personajes dialogan, y el montaje está hecho de tal manera que vemos en pantalla la cara de quien habla como si fuéramos su interlocutor, y el montaje pasa de un rostro al otro cada vez que uno de los personajes toma la palabra. De esta manera, estamos en el lugar del interactuante en el dialogo, como si el personaje se estuviera dirigiendo al espectador, en lugar de al otro personaje.
} 
historia personal del artista está en juego en nuestra comprensión e involucramiento con la obra. En otros casos, no es la identidad ni la historia del artista, sino su acción material concreta que dio lugar a la creación de la obra la que nos resulta importante. Como sostiene Gell (1998/2016) en ocasiones la admiración que nos produce una obra se funda en que quedamos maravillados por la maestría en la agencia del autor al hacer algo que nosotros no podríamos hacer (con un instrumento musical, con la voz, con las manos al dibujar o pintar o esculpir, con el cuerpo al danzar, o al actuar, etc.). Asimismo, hay aspectos de la materialidad de la obra que son huellas de la presencia de un acto humano (por ej. la pincelada o el trazo en un dibujo, etc.), y al percibir el trazo captamos la calidad del movimiento corporal que lo generó. Gallese $(2010,2017)$ muestra que en estos casos se activan además del córtex visual, el sistema de neuronas espejo, e interpreta este hecho afirmando que está en proceso una tarea de simulación corporizada de la acción del artista sobre el lienzo. Independientemente de que sea necesario leer así la activación de las neuronas espejo en estos casos, ${ }^{10}$ lo cierto es que la visión del producto de una acción humana nos remite a la acción humana que le dio existencia y por lo tanto encarna intenciones y emociones del agente. Estos rastros materiales de la agencia humana son justamente los rasgos expresivos que activan nuestras atribuciones psicológicas en el contexto de nuestra interacción de segunda persona con nuestros congéneres.

En quinto lugar, se puede pensar que la obra misma - el objeto o evento material - es una segunda persona. Al fin y al cabo la empatía buscaba atribuir (proyectar) sentimientos en la obra (el cuadro, los sonidos) y Gell (1988/2016) considera que el objeto mismo tiene agencialidad propia, independiente de las agencialidades humanas que dieron lugar a su existencia. Más aún, la obra en consideración podría no ser mimética, ni ficcional, ni narrativa, ni representativa, sino ser completamente abstracta, como un cuadro de Rothko o de Pollock. Una pregunta que quedará pendiente es en qué medida la obra misma tiene una interacción de segunda persona con el apreciador que sea diferente de las que este tiene con cada una de las "personas" involucradas mencionadas en los puntos anteriores. Parece haber consenso en la idea de que hay formas de interacción con las obras que de alguna manera se asemejan a las formas en las que los humanos interac-

\footnotetext{
${ }^{10}$ Gallese 2005 sugiere que una lectura posible del rol de la activación del sistema de neuronas espejo (dado que se activan de la misma manera en el caso de la primera que de la tercera persona -es decir no distinguen a yo de otro) es pensarlas como codificando acciones tipo, en lugar de ser los correlatos neurales de los pensamientos particulares acerca de la propia acción o de la acción ajena. Esta lectura del rol del sistema de las neuronas espejo vuelve compatible estos hallazgos con la perspectiva de segunda persona, en la medida en que codificarían tipos de acciones intencionales, es decir, conceptos psicológicos, en lugar de atribuciones particulares de estados psicológicos a sujetos (yo u otro). No tengo espacio para desarrollar en detalle esta idea en este trabajo.
} 
tuamos entre nosotros, al dotar a la obra de afectividad y agencialidad.

En sexto lugar, para dar cuenta cabal de nuestra comprensión e involucramiento con las obras de arte podríamos considerar las relaciones de segunda persona que tenemos los apreciadores entre nosotros. ${ }^{11}$ Como dije, arriba, hay formas de recepción del arte muy pasivas, pero hay otras mucho más activas, en las que las interacciones entre los espectadores es esencial para la constitución de la experiencia estética. Pensemos en una fiesta electrónica o en un cineclub, en el que la calidad de la experiencia relativa a la película considerada varía en función de los comentarios y discusiones que los diversos aspectos de la obra suscitan en un grupo de espectadores, que ponen sus ideas e impresiones en común. Excepto que estemos solos, como cuando leemos un libro o vemos una película en streaming en la soledad de nuestro hogar, los otros cerca nuestro que se sobresaltan o sonríen con nosotros, aun en la clásica sala de cine o teatro, colaboran en la constitución de nuestra experiencia estética. Muchos fenómenos de segunda persona, como el contagio emocional, son parte de nuestra experiencia con otros ante las obras de arte.

De acuerdo con la perspectiva de segunda persona que presenté en el apartado anterior, las interacciones entre seres humanos suelen involucrar una mu- tua comprensión como seres con mentes, con experiencias, emociones, intenciones, etc., que se muestran en la corporalidad del otro en la interacción. En efecto, una minúscula contracción de un músculo en la cara de nuestro interlocutor nos indica que el otro no está de acuerdo con lo que decimos, o que le da asco o risa nuestra conducta; una sutil desviación de su mirada nos muestra que no nos está prestando atención; una inclinación de su cuerpo puede mostrarnos su deseo de alcanzar algo; una mirada puede decir más que mil palabras, dice el proverbio. Y esta comprensión implícita, automática, directa, de las mentes ajenas está en juego en cada una de las interacciones mencionadas arriba, en la interacción entre los personajes que captamos como espectadores, en las interacciones entre los artistas, por ej. entre los músicos al ejecutar en conjunto ante nosotros una pieza, en nuestra comprensión y reacción ante los rasgos materiales en la obra de la agencialidad humana que la creó, en nuestro involucramiento con los personajes, por ejemplo en una película cuando un personaje mira a cámara y se dirige a la audiencia rompiendo la cuarta pared, o en la típica escena de dialogo entre dos personajes en los que la cámara nos pone en el lugar del interlocutor que está escuchando al otro, y alterna la perspectiva de cada uno de los personajes a medida que el dialogo se desarrolla, pa-

\footnotetext{
${ }^{11}$ Agradezco a María Marchiano la sugerencia.
} 
ra involucrarnos en ese intercambio cara a cara entre los personajes. Lo importante a resaltar es que pueden no estar todas estas situaciones presentes en la misma obra, pero en general hay más de una de ellas en juego en nuestra apreciación de la obra. Las distintas interacciones con personajes, performers, artistas, etc. se superponen en nuestra experiencia con la obra. Así, hay una pluralidad de agentes reales o ficticios, percibibles directamente o inferidos a partir de aspectos diversos de la obra ${ }^{12}$, con los que interactuamos simultánea o sucesivamente al apreciar una obra de arte. 13

Así, parece extraño suponer que deba haber una coincidencia entre la mente del apreciador y alguna otra mente, que un único estado mental (el del apreciador) deba proyectarse en la obra. Por el contrario, parece que la comprensión de todos estos elementos está en juego en la experiencia estética. En mi opinión el apreciador tiene que hacer un trabajo muy arduo para acceder a los estados mentales relevantes de todos estos agentes en juego para tener una experiencia estética, y la cualidad de esa experiencia no tiene el por qué ser idén- tica a la de nada que hubo en juego allí para llegar a ella.

Es importante destacar que la comprensión e involucramiento con los diferentes "otros" que son responsables de nuestra experiencia ante la obra no alcanzan para entender la experiencia estética, así como las atribuciones de segunda persona solas no alcanzan para dar cuenta de las atribuciones psicológicas humanas. Se necesita incorporar otros elementos que nada tienen que ver con la perspectiva de segunda persona para comprender cabalmente nuestra experiencia ante las obras de arte. La perspectiva de segunda persona nos provee de herramientas para pensar en aspectos de nuestra interacción con las diversas "personas" involucradas en las obras, así como remarcar que las respuestas emocionales del apreciador son parte de la experiencia estética que puede no coincidir con los estados emocionales de ninguno de los elementos de la obra.

Así, la alternativa más interesante, para mi, es la de pensar que la relación del apreciador con estos agentes es la de una interacción de segunda persona, o surgida a partir de experiencias de se-

\footnotetext{
${ }^{12}$ Me refiero al narrador implícito que es "construido" o "inferido" a partir de la obra, tal como sostuve arriba.

${ }^{13} \mathrm{La}$ experiencia ante el arte tiene siempre una dimensión temporal, como me señaló Isabel Martínez en conversación. En el caso de las artes temporales, como la música o la danza o el cine, la obra misma de desarrolla temporalmente, y nuestra experiencia ante ella no puede sino ser temporal, acompañando el desarrollo de la obra. En el caso de la pintura o escultura, si bien el objeto no se desarrolla temporalmente (aunque existe a través del tiempo), somos nosotros, en nuestra forma de apreciarla, los que generamos este desarrollo temporal de la experiencia. En efecto, cuando apreciamos una pintura o escultura en un museo nos movemos, "escaneamos" con la mirada diversos aspectos de la obra, nos alejamos y acercamos a ella, es decir, construimos una experiencia que dura en el tiempo, no es instantánea, y que nos permite acceder a diversos detalles de la obra a medida que la apreciamos desde diversos ángulos. Este desarrollo temporal a su vez nos permite captar esta diversidad de elementos que constituyen la obra, e integrarlos para generar experiencias sucesivamente más complejas. Agradezco a I. Martínez sus comentarios acerca de este punto.
} 
gunda persona, en donde no hay identidad sino una apropiada reactividad emocional y cognitiva ante los elementos en juego/agentes. Y que la experiencia estética surge a partir de estas múltiples interacciones de segunda persona - de los personajes entre sí, de nosotros - los apreciadores - con los personajes, con la situación, con el narrador, con el autor, etc. - más una enorme cantidad de otros elementos relevantes: la experiencia pasada del sujeto, la información que posee -del mundo en general, de la forma de arte particular, del período artístico, de la autora, etc.-, su trasfondo cultural, sus capacidades perceptivas (auditivas para la música, visuales para la pintura, tal vez también táctil en este caso...), las habilidades linguísticas para comprender los textos literarios y guiones cinematográficos, etc. Como es evidente, no todas estas habilidades cognitivas están presentes en la constitución de nuestra experiencia ante todas las obras de arte. Las formas de arte son muy diversas: algunas obras de arte son objetos materiales, otras eventos sonoros, algunas suponen la visión y la audición, alguna involucran cuerpos en movimiento, otras no, algunas suponen nuestra capacidad de comprender narraciones, algunas suponen conocer ciertos simbolismos propios de una cultura o tradición, conocer el momento histórico en el que se crearon, o al que se refieren, etc. Asimismo, las capacidades cognitivas, la información previa, las experiencias anteriores de cada uno de los apreciadores, los valores y creencias que posee, son todos elementos que se pondrán en juego en cada una de las experiencias individuales que en los diversos momentos de nuestras vidas tenemos con las diversas obras. Nunca nos bañamos dos veces en el mismo río, nunca surge la misma experiencia estética ante una misma obra, nunca repetimos de manera invariable formas de interacción con los otros. Cada particular relación con una obra involucra algunos de estos elementos mencionados y no otros, o un mayor o menor desarrollo de alguna de estos aspectos, y por tanto genera diversa formas de comprensión e involucramiento con las obras de arte. No estoy en posición, ni hay espacio suficiente en este trabajo, para desarrollar en detalle todos estos diversos aspectos que nos permiten constituir las diferentes experiencias estéticas que somos capaces de disfrutar. El objetivo que me propuse en este trabajo, y que espero haber logrado, era mucho más modesto: presentar un argumento a favor de reemplazar la empatía y la simulación por la perspectiva de segunda persona como un trampolín más apropiado para dar cuenta de nuestra comprensión e involucramiento con el arte.14

\footnotetext{
${ }^{14}$ Agradezco los comentarios de Tomás Balmaceda, Fernando Broncano, Jesica Buffone, Federico Burdman, Silvia Español, Diego Lawler, Eleonora Orlando, María Marchiano, Isabel C. Martínez, Matías Tanco, Marina Trakas y Jesús Vega a una versión previa de este trabajo.
} 


\section{Bibliografía}

BRINCK, I. y REDDY, V. (2020) "Dialogue in the making: emotional engagement with materials". Phenomenology and the Cognitive Science. 19: 23-45.

COPLAN, A. y GOLDIE, P. (2011) Empathy. Philosophical ans Psychological Perspectives. OUP.

CURRIE, G. (1995) "Imagination as simulation: Aesthetics meets cognitive science". In DAVIES, Martin STONE, Tony (eds.), Mental Simulation. Blackwell (1995).

CURRIE, G. RAVENSCROFT, I. (2002) Recreative Minds: Imagination in Philosophy and Psychology. OUP.

DE JAEGHER, H. DI PAOLO, E. (2007) "Participatory sense-making”. Phenomenology and Cognitive Science 6: 485-507.

DISSANAYAKE, E. (2000). "Antecedents of the temporal arts in early mother-infant interaction". En N. L. Wallin, B. Merker y S. Brown (Eds.). The Origins of Music. Cambridge, MA: The MIT Press.

ESPAÑOL, S. (2014). La forma repetición variación. Una estrategia para la reciprocidad. En S. Español (comp.) Psicología de la música y del desarrollo. Una exploración interdisciplinaria sobre la musicalidad humana (pp. 157-192). Buenos Aires: Paidós.

ESPAÑOL, S. y PÉREZ, D. "Los juegos, los contrastes, las artes”. Boletín de Estética, 33, 2015, pp- 5-40. ISSN 2408-4417

FREEDBERG, D. and GALLESE, V. (2007) "Motion, emotion and empathy in esthetic experience" TRENDS in cognitive science, Vo. 11, no. 5: 197:203

GAllagher, S. (2001). "The Practice of Mind: Theory, Simulation or Interaction”. En E. Thompson (Ed.). Between Ourselves. Second-person Issues in the Study of Conciousness. Imprint Academic, pp. 83-108.

GALLAGHER, S. (2007) "Logical and phenomenological arguments against simulation theory". In HUTTO, D. RATCLIFFE, M. (eds.) Folk Psychology Re-assessed. Springer, pp. 63-78.

GALLESE, V.; FADIGA, L.; FOGASSI, L.; RIZZOLATTI, Giacomo (1996). "Action recognition in the premotor cortex". Brain. 119 (2): 593-609.

GALLESE, V., GOLDMAN, A. "Mirror neurons and the simulation theory of mind-reading". Trends Cogn. Sci. 1998 Dec 1; 2(12): 493-501. doi: 10.1016/s1364-6613(98)01262-5. PMID: 21227300.

GALLESE, V. (2005) "Embodied simulation: From neurons to phenomenal experience" Phenomenology and the Cognitive Sciences (2005) 4: 23-48.

GALLESE, V. (2010) "Mirror neurons and art" BACCI, F. MELCHER, D. (eds.) Art and the Senses. OUP, Oxford.

GALLESE, V. (2017) "Visions of the body. Embodied simulation and aesthetic experience" Aisthesis 10 (1): 41:50.

GELL, A. (1998/2016) Arte y Agencia. Una teoría antropológica. SB Editorial, Buenos Aires.

GOLDMAN, A. (1993) "The Psychology of Folk Psychology". Behavioral and Brain Sciences 16 (1):15.28.

GOLDMAN, A. (2006) Simulating Minds. OUP, Oxford.

GOMILA, A. (2001) "La perspectiva de segunda persona: mecanismos mentales de la intersubjetividad". Contrastes 6: 65-86.

GOMILA, A. (2009) "Musical expression and the second person perspective". In ALVAREZ, I.; PÉREZ, F. PÉREZ, H. (eds.) Expression in the performing arts. Cambridge Scholars Publishing, pp. 66-85.

GOMILA, A. (2013) "Las emociones en el teatro: ¿por qué nos involucramos emocionalmente con una representación?" In SORIA TOMÁS, G. (ed.) La representación de las pasiones: perspectivas artísticas, filosóficas y científicas. Madrid: Dykinson, pp. 57-78.

LIPPS, T. (1924) Fundamentos de la estética. Ed. D. Jorro, Madrid.

MALLOCH, S. y TREVARTHEN, C. (2009) "Musicality: Communicating the vitality and interests of life". En S. MALLOCH y C. TREVARTHEN, C. (Eds). Communicative musicality: Exploring the basis of human companionship. Oxford: Oxford University Press, pp. 1-11.

MARTÍNEZ, I.C., ESPAÑOL, S.A. PÉREZ, D.I. "The Interactive Origin and the Aesthetic Modelling of Image-Schemas and Primary Metaphors" Integrative Psychological and Behavioral Science 2018 Dec; 52(4): 646-671.

MATíNEZ, I.C. (2021) Dossier. La segunda persona y la música. El oído pensante. Vol. 9 Núm. 2 (2021): Septiembre 2021 - Febrero 2022: 9-158.

PÉREZ, D. GOMILA, A. (2021). The second person: Social cognition in human interactio. London: Routledge.

PÉREZ, D. (2018a) "Filosofia, Ciencias Cognitivas y Sentido Común: el caso de la segunda persona de la atribución mental", Revista de Filosofia Moderna e Contemporanea, Brasilia, v.6, n.1, p. 49-72 49 ISSN: 2317-9570.

PÉREZ, D. (2018b) "A expressão das emoções e a segunda pessoa (ou quanto podemos saber da mente do outro através de sua expressão)", Debates contemporaneos em filosofia da mente. Leal Toledo, G. Gouvea, R y Alves, M. (eds.) Editorial FiloCzar, San Pablo, Brasil, 2018, pp. 19-94.

PÉREZ, D. (2014) "Conceptos de primera persona" en SMITH, P. J.; SILVA FILHO, W. J.; SANTOS, P. (orgs.), Crença, verdade, racionalidade: ensaios de filosofia analítica, Salvador: EDUFBA, pp. 15-36 .

PÉREZ, D. (2013). Sentir, desear, creer. Una aproximación filosófica a los conceptos psicológicos. Buenos Aires: Prometeo. 
PÉREZ, D. (2011) "Phenomenal concepts, color experience, and Mary's puzzle", Teorema. XXX/3 pp. 113-133. ISSN $0210-1602$.

REDDY, V. (2008). How infants know mind. London: Harvard University Press.

RIZZOLATTI, G.; FADIGA, L.; GALLESE, V.; FOGASSI, L. (1996). "Premotor cortex and the recognition of motor actions". Cognitive Brain Research. 3 (2): 131-141.

SCOTTO, C. (2002) "Interacción y atribución mental: la perspectiva de la segunda persona”. Análisis Filosófico 22(2): $135-151$.

STERN, D. (2010). Forms of vitality. Exploring dynamic experience in psychology, the arts, psychotherapy and development. New York: Oxford University Press.

WALTON, K. (2015) In Other Shoes. Music, Metaphor, Empathy, Existence. OUP.

Recibido: 02/02/2021

Aprobado: $12 / 03 / 2021$

Publicado: 30/04/2021 
